\title{
Anatomical substrate and scalp EEG markers are correlated in subjects with cognitive impairment and Alzheimer's disease
}

\section{Davide V. Moretti*, Giovanni B. Frisoni, Giuliano Binetti and Orazio Zanetti}

Istituto Di Ricovero e Cura a Carattere Scientifico S. Giovanni di Dio Fatebenefratelli, Brescia, Italy

\section{Edited by:}

Mark A. Smith, Case Western Reserve

University, USA

\section{Reviewed by:}

Kerry Coburn, Mercer University

School of Medicine, USA

Leslie Prichep, New York University

School of Medicine, USA

${ }^{*}$ Correspondence:

Davide V. Moretti, Via Pilastroni, 4

25125 Brescia, Italy

e-mail:davide.moretti@afar.it
Dementia is a syndromic diagnosis, encompassing various stage of severity and different anatomo-physiological substrates. The hippocampus is one of the first and most affected brain regions affected by both Alzheimer's disease (AD) and mild cognitive impairment (MCI). Moreover, chronic cerebrovascular disease (CVD) is one of the major risk factor for developing dementia. Recent studies have demonstrated different relationship between the anatomical substrate and scalp electroencephalography (EEG) markers. Indeed, modifications of EEG rhythmicity is not proportional to the hippocampal atrophy, whereas changes in EEG activity are directly proportional to the load of subcortical CVD. The computation of the EEG spectral power and the analysis of the functional coupling of brain areas, through linear coherence, are two of the most known processing methods in EEG research. Two specific EEG markers, theta/gamma and alpha3/alpha2 frequency ratio have been reliable associated to the atrophy of amygdalo-hippocampal complex. Moreover, theta/gamma ratio has been related to $\mathrm{MCl}$ conversion in dementia and alpha3/alpha2 ratio has been specifically related to $\mathrm{MCl}$ conversion in AD. The functional coupling of brain areas is also modulated by hippocampal atrophy. In the $\mathrm{MCl}$ subjects, hippocampal atrophy is linked to an increase of interhemispheric coherence seen on frontal and temporal regions whereas subcortical CVD is linked to a decrease of coherence in fronto-parietal regions. In the present study the most significant results of recent studies on correlation between scalp EEG, cognitive decline, and anatomical substrate have been reviewed, with particular attention to the relationships between EEG changes and hippocampal atrophy. The following review is not intended to provide a comprehensive summary of the literature. Rather it identifies and discusses selected studies that are designed to find the specific correlation between scalp EEG markers and anatomo-pathological substrate. The principal aim is to propose a plausible neurophysiological theoretical model of the cognitive decline as mirrored by both structural and functional tools of research.

Keywords: EEG markers, Alzheimer's disease, mild cognitive impairment, alpha rhythm, theta rhythm

\section{INTRODUCTION}

The hippocampus is one of the first and most affected brain regions impacted by both Alzheimer's disease (AD) and mild cognitive impairment (MCI; Arnold et al., 1991; Bobinski et al., 1995; Price and Morris, 1999; Bennett et al., 2004; Schonheit et al., 2004). In mild-to-moderate AD patients, it has been shown that hippocampal volumes are $27 \%$ smaller than in normal elderly controls (Callen et al., 2001; Du et al., 2001), whereas patients with MCI show a volume reduction of $11 \%$ (Du et al., 2001). So far, from a neuropathological point of view, the progression of disease from early or very early MCI to later stages seems to follow a linear course. Nevertheless, there is some evidence from functional (Gold et al., 2000; Della Maggiore et al., 2002; Hamalainen et al., 2006) and biochemical studies (Lavenex and Amaral, 2000) that the process of conversion from non-demented to clinically evident demented state is not so linear. Recent fMRI studies have suggested increased medial temporal lobe (MTL) activations in MCI subjects vs controls, during the performance of memory tasks (Dickerson et al., 2004, 2005). Nonetheless, fMRI findings in MCI are discrepant, as MTL hypoactivation similar to that seen in $\mathrm{AD}$ patients (Pariente et al., 2005) has also been reported (Machulda et al., 2003). Recent postmortem data from subjects - who had been prospectively followed and clinically characterized up to immediately before their death - indicate that hippocampal choline acetyltransferase levels are reduced in Alzheimer's dementia, but in fact they are upregulated in MCI (Lavenex and Amaral, 2000), presumably because of reactive upregulations of the enzyme activity in the unaffected hippocampal cholinergic axons. Quantitative electroencephalography (EEG) have been demonstrated a reliable tool in identifying specific patterns in dementia research (John and Prichep, 1990; Prichep et al., 1994; Coburn et al., 2006; John and Prichep, 2006). Previous EEG studies (Jelic et al., 1996, 2000; Babiloni et al., 2000; Ferreri et al., 2003) have shown a decrease - ranging from 8 to $10.5 \mathrm{~Hz}$ (low alpha) - of the alpha frequency power band in MCI subjects, when compared to normal elderly controls (Zappoli et al., 1995; Huang et al., 2000; Jelic et al., 2000; Koenig et al., 2005; Babiloni et al., 2006). However, a recent study has shown an increase - ranging from 10.5 to $13 \mathrm{~Hz}$ (high alpha) - of the alpha frequency power band, on the occipital region in MCI subjects, when compared to normal elderly and AD patients (Babiloni et al., 2006). These somewhat 
contradictory findings may be explained by the possibility that MCI subjects have different patterns of plastic organization during the disease, and that the activation (or hypoactivation) of different cerebral areas is based on various degrees of hippocampal atrophy. If this hypothesis is true, then EEG changes of rhythmicity have to occur non-proportionally to the hippocampal atrophy, as previously demonstrated in a study of auditory evoked potentials (Golob et al., 2007).

\section{HIPPOCAMPAL ATROPHY AND EEG MARKERS}

A recent study (Moretti et al., 2007), has confirmed the hypothesis that the relationship between hippocampal volume and EEG rhythmicity is not proportional to the hippocampal atrophy, as revealed by the analyses of both the relative band powers and the individual alpha markers. Such a pattern seems to emerge because, rather than a classification based on clinical parameters, discrete hippocampal volume differences (about $1 \mathrm{~cm}^{3}$ ) are analyzed. Indeed, the group with moderate hippocampal atrophy showed the highest increase in the theta power band on frontal regions, and of the alpha 2 and alpha3 power bands on frontal and temporo-parietal areas (Figures 1-3).

Recently, two specific EEG markers, theta/gamma and alpha3/ alpha2 frequency ratio have been reliable associated to the atrophy of amygdalo-hippocampal complex (AHC; Moretti et al., 2009a,b), as well as with memory deficits, that are a major risk for the development of AD in MCI subjects (Figure 4). Based on the tertile values of decreasing $\mathrm{AHC}$ volume, three groups of $\mathrm{AHC}$ increasing amount of atrophy were obtained. AHC atrophy is associated with memory deficits as well as with increase of theta/gamma and alpha3/alpha2 ratio. Moreover, when the amygdala and hippocampal volume are separately considered, within AHC, the increase of theta/gamma ratio is best associated with amygdala atrophy whereas alpha3/ alpha2 ratio is best associated with hippocampal atrophy.

A large body of literature has previously demonstrated that in subjects with cognitive decline is present an increase of theta relative power (Moretti et al., 2007, 2008a,b), a decrease of gamma relative power (Stam et al., 2003; Moretti et al., 2008a,b) as well as an increase of high alpha as compared to low alpha band (Moretti et al., $2008 \mathrm{a}, \mathrm{b})$. On the whole theta/gamma ratio and alpha3/alpha2 ratio could be considered reliable EEG markers of cognitive decline.

The amygdalo-hippocampal network is a key structure in the generation of theta rhythm. More specifically, theta synchronization is increased between lateral amygdala and CA1 region of hippocampus during long-term memory retrieval, but not during short-term or remote memory retrieval (Seidenbecher et al., 2003; Narayanan et al., 2007). In particular, the AHC is critically involved in the formation and retention of fear memories (Narayanan et al., 2007). Theta synchronization in AHC appears to be a neural correlate of fear, apt to improve the neural communication during memory retrieval (Narayanan et al., 2007). On the other hand, the retrieval of hippocampus-dependent memory is provided by the integrity of CA3-CA1 interplay coordinated by gamma oscillations (Montgomery and Buzsaki, 2007). Our results confirm and extends all previous findings. The atrophy of AHC determines increasing memory deficits. The brain oscillatory activity of this MCI state is characterized by an increase of theta/gamma and alpha3/alpha2 relative power ratio, confirming the overall reliability of these EEG markers in cognitive decline. Previous results suggest that theta synchronization is mainly due to the amygdala activation or as a subsequent final net effect within the AHC, driven by the amygdala

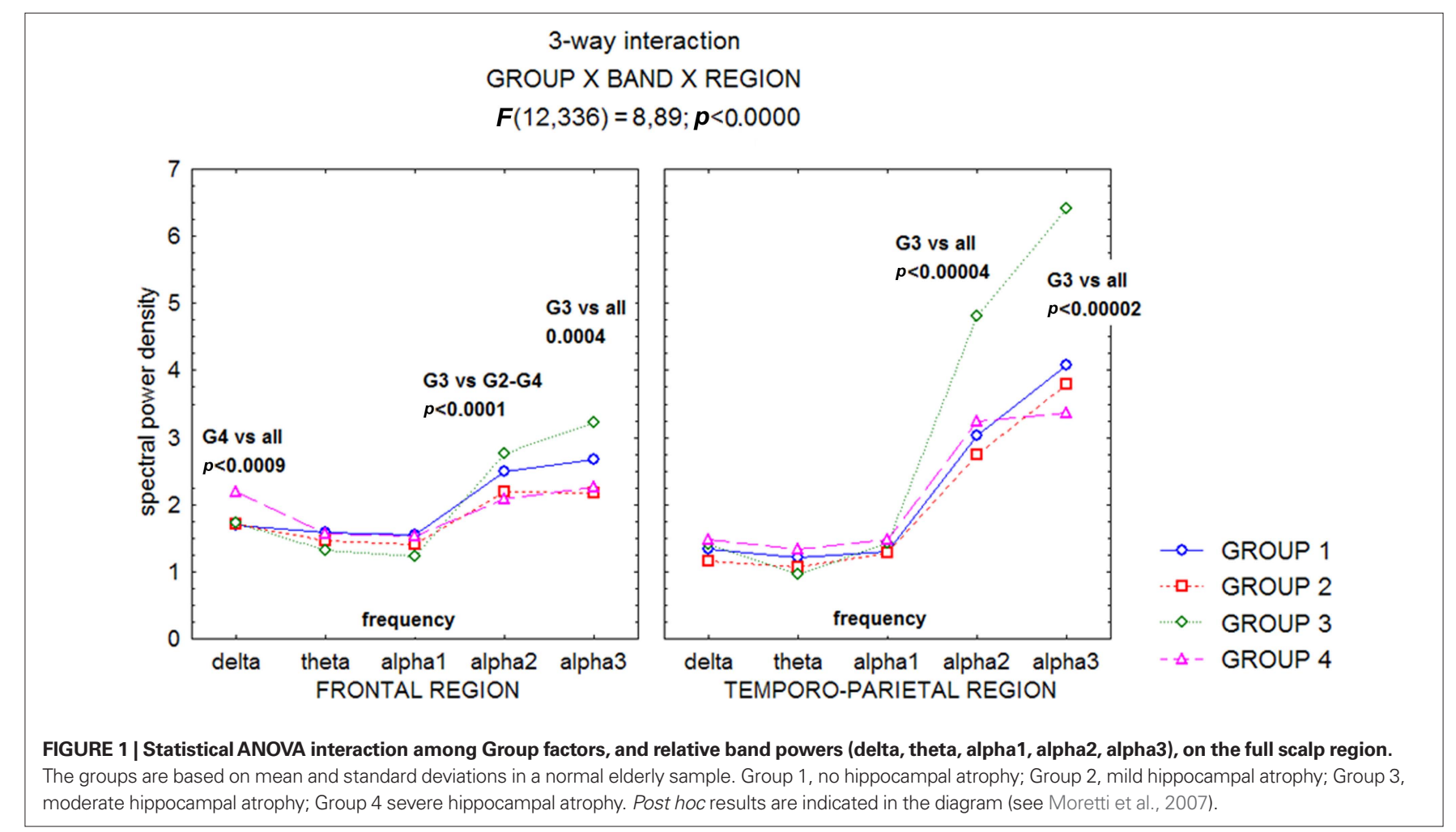



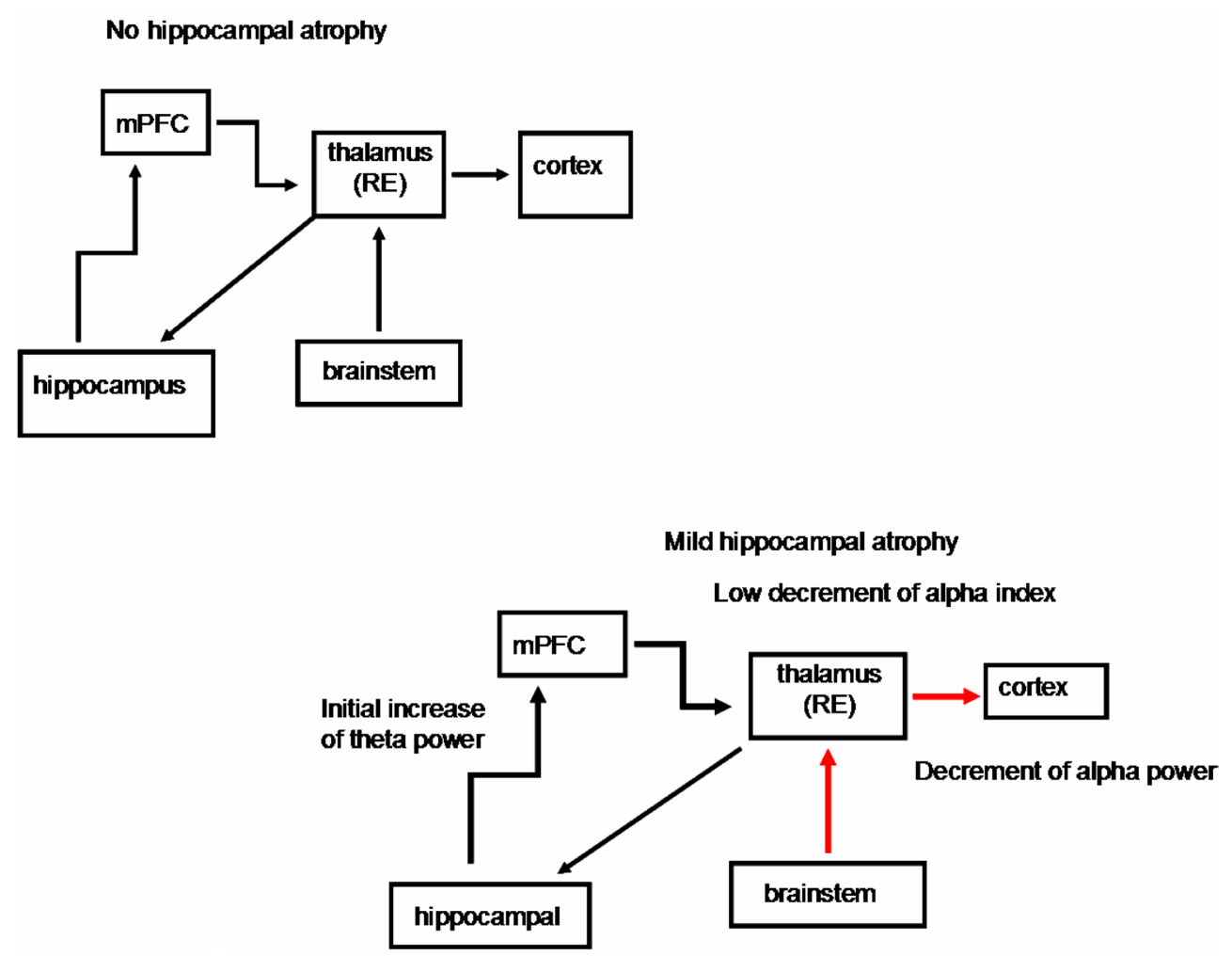

FIGURE 2 | Schematic diagrams of the possible structures involved in the thalamo-cortical arrhythmia in groups without hippocampal atrophy and with mild hippocampal atrophy. The EEG changes in the mild hippocampalatrophy group are probably due to a prevalent depolarizing effect of the brainstem cholinergic system on the thalamus, because of initial neuronal loss in the cholinergic basal forebrain. On the other hand, the initial hippocampal atrophy gives rise to an increase in the theta activity, but it is not able to trigger thalamo-cortical synchronization activity (see Moretti et al., 2007). mPFC, medial prefrontal cortex; thalamus RE, nucleus reuniens of the thalamus. Black thick arrow, excitatory activity; blue arrow, synchronization effect; red arrow, desynchronization effect. excitation. The increase in theta activities in AHC, is considered as an increase in neuronal communication apt to promote or stabilize synaptic plasticity (Montgomery and Buzsaki, 2007). This is in relation to the effort to retention associative memories (Sauseng et al., 2004), and could be active also during an ongoing degenerative process. The excitation mechanism could be facilitated by the loss of GABA inhibitory process, determining the decrease of gamma rhythm generation in AD (Bragin et al., 1995; Montgomery and Buzsaki, 2007).

\section{HIPPOCAMPAL ATROPHY AND FUNCTIONAL COUPLING OF CORTICAL BRAIN AREAS}

The functional coupling of brain areas is also modulated by hippocampal atrophy. In the MCI subjects, hippocampal atrophy is linked to an increase of interhemispheric coherence seen on frontal and temporal regions whereas subcortical cerebrovascular disease (CVD) is linked to a decrease of coherence in frontoparietal regions. Moreover, significant differences of EEG functional coupling were present in the fronto-temporal network in MCI patients with severe CVD and severe hippocampal atrophy, but with a different pattern. In high CVD, the EEG coherence in low frequencies was increased (with the exception of alpha1 band) while coherence in the fast frequencies was decreased in a way directly proportional to increasing damage. In high hip- pocampal atrophy a change of coherence was present in the delta and alpha 2 frequency bands that was not proportional to the hippocampal damage, fast frequencies being unaffected. Moreover, our results show a lateralization (right hemisphere for CVD and left hemisphere for hippocampal atrophy) of the pathological modifications of functional coupling (Moretti et al., 2008a,b) along the course of the disease.

An increase of neuronal excitability could explain the pathological modifications of the functional coupling (Moretti et al., 2008a). When MCI subgroups are considered, based on different pathological substrate, the results show that the increase of interhemispheric coherence seen in frontal (delta frequency) and temporal regions (delta, theta, alpha1, and, in a tendential way, alpha2 and alpha3 frequency bands) is exclusively linked to the hippocampal atrophy (Figure 5). Previous studies show that the increase of coherence between temporal regions is determined by an increase of excitability (Ferreri et al., 2003; Vecchio et al., 2007). This hypothesis could receive a support from studies demonstrating a dysregulation of inhibitory GABA-ergic system following the hippocampal atrophy (De Curtis and Parè, 2004; Gloveli et al., 2005). Through the hippocampal commissure, the increase of excitability could spread over the two hemispheres. Our results confirm this view because the increase of coherence between temporal regions is present only in the MCI subjects with the greater hippocampal atrophy. 


\section{Moderate hippocampal atrophy}

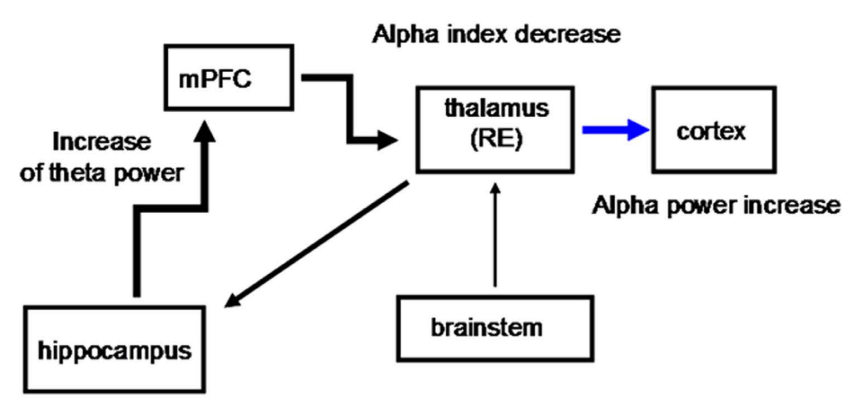

Severe hippocampal atrophy

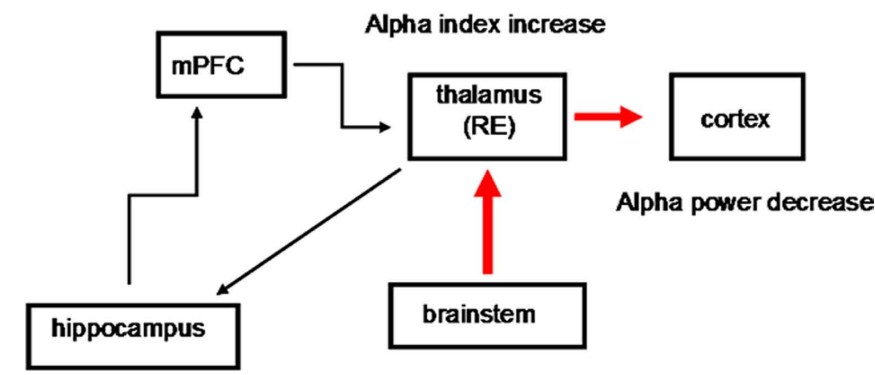

FIGURE 3 | Schematic diagrams of the possible structures involved in the thalamo-cortical arrhythmia in groups with moderate and severe hippocampal atrophy. The progressive hippocampal atrophy, as in the moderate hippocampal-atrophy group, triggers thalamo-cortical synchronization, with increase in the alpha power band. This is likely to happen because the return pathway from the cortex to the hippocampus is not direct, but it mainly relays to the midline and mediodorsal thalamic nuclei. The decrease in the values for the alpha frequency markers in the moderate hippocampal-atrophy group also suggests a greater hyperpolarization state of thalamo-cortical pathways. As the hippocampal atrophy progresses, like in the group with severe hippocampal atrophy, the thalamo-cortical activity is sustained not by cortical activation, but by the prevailing cholinergic desynchronizing activity of the brainstem, with a decrease in the relative alpha power but the highest values for the alpha indices (see Moretti et al., 2007). mPFC, medial prefrontal cortex; thalamus RE, nucleus reuniens of the thalamus. Black thick arrow, excitatory activity; blue arrow, synchronization effect; red arrow, desynchronization effect.
Precedent literature (Jiang, 2005; Jiang and Zheng, 2006) showed an increase of coherence in MCI subjects as compared to normal controls during a working memory task, in which prefrontal and medial temporal areas are involved (Johnson, 2006). Our results suggest that it could be due to an increase of excitability specifically due to the hippocampal atrophy. A decrease of fronto-parietal coherence characterized MCI with hippocampal atrophy. Given the absence of either a significative CVD or cholinergic vascular damage in this group of MCI compared to normal old subjects, a possible explanations is that the increase of excitability in medial temporal areas subsequent to the hippocampal atrophy creates a sort of "temporal areas wall" impairing the long-range, frontoparietal functional connections within each hemispheres. A recent study (Zheng et al., 2007) has demonstrated that MCI subjects have a decrease in intrahemispheric and an increase in interhemispheric coherence in alpha1 and alpha2 frequency bands during an experimental condition needing a memory demand increase in order to maintain a good level of cognitive processing. Our results suggest that this compensatory effort is more frequently observed in subjects with hippocampal atrophy. Moreover, it could be argued that this activity (Pijnenburg et al., 2004) emerges as a new default mode of brain activity (Moretti et al., 2007) characterized by an hyperexcitability of the cortex, even at a rest state. No difference was found on frontal and parietal interhemispheric coherence between normal old and MCI subjects with hippocampal atrophy. This could suggest that, in the initial stage of disease, interhemispheric connections are damaged only on the temporal lobes. Another possible explanation is that the hippocampal functional connections are mostly branched within each hemisphere (De Curtis and Parè, 2004; Gloveli et al., 2005).

\section{HIPPOCAMPAL ATROPHY, COGNITIVE DEFICITS (MEMORY AND ATTENTION), AND EEG ACTIVITY}

The vulnerability and damage of the connections of hippocampus with amygdala could affect reconsolidation of long-term memory and give rise to memory deficits and behavioral symptoms. Several experiments shows that amygdala activity is prominent during period of intense arousal, e.g., the anticipation of a noxious stimulus (Parè et al., 2002) or the maintenance of vigilance to negative stimuli (Garolera et al., 2007). So far, the theta synchronization induced by the amygdala is deeply involved in endogenous attentional mechanism. Interestingly, the increase of high alpha synchronization has been found in internally cued mechanisms of attention, associated with inhibitory top-down processes (Klimesch et al., 2007). Of note, the amygdala is intimately involved in the anatomo-physiological anterior pathways of attention through its 


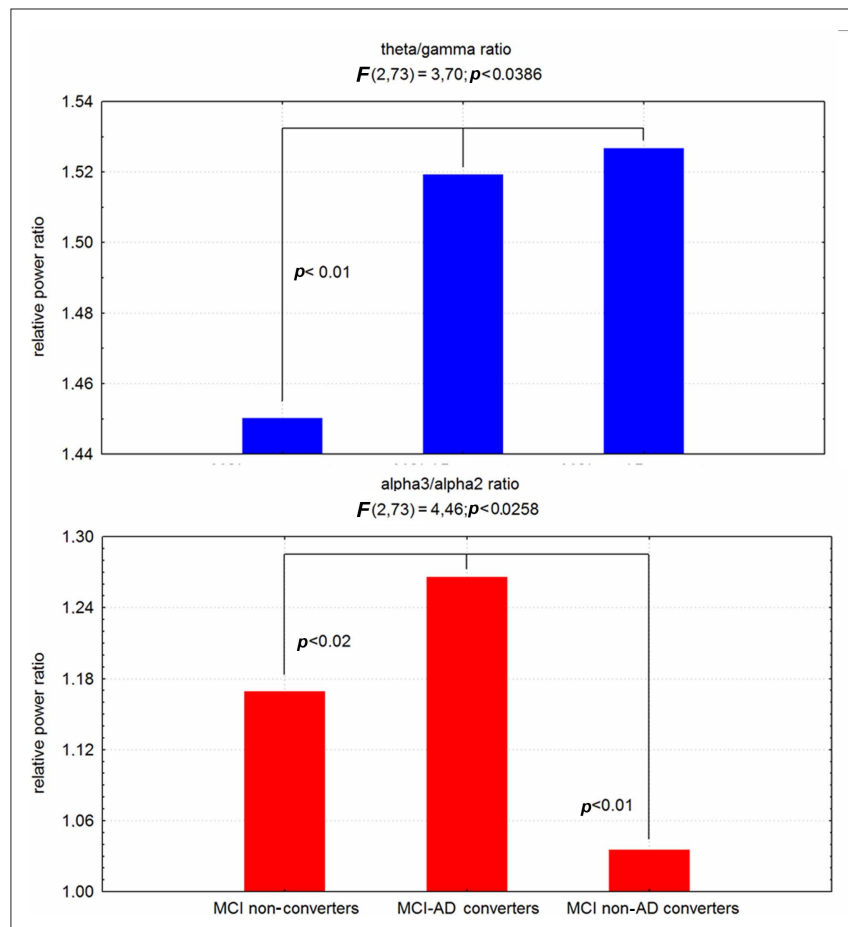

FIGURE 4 |ANOVA results of theta/gamma and alpha3/alpha2 relative power ratio. In the graph post hoc results are shown. connections with anterior cingulated cortex, anteroventral, anteromedial, and pulvinar thalamic nuclei (Young et al., 2007). The particular role of amygdala in negative human emotions could indicate that AHC atrophy is associated with excessive level of subcortical inputs not adequately filtered by attentive processing, determining fear and anxiety, and generating cognitive interference in memory performance. Of note, an altered emotional response is very frequent in MCI patients (Ellison et al., 2008; Rozzini et al., 2008). In a feed-back process, this alteration could determine a general state of "hyperattention" during which top-down internal processes prevail on the bottom-up phase, altering attention mechanism, and preventing a correct processing of sensory stimuli. Focused attention has been found impaired in MCI patients in particular when they have to benefit from a cue stimulus (Johannsen et al., 1999; Berardi et al., 2005; Levinoff et al., 2005; Tales et al., 2005a,b). This particular state could be useful for maintain a relatively spared global cognitive performance, whereas it could fail when a detailed analysis of a sensory stimulus is required. This "hyperattentive" state could represent the attempt to recollect memory and/or spatial traces from hippocampus and to combine them within associative areas connected with hippocampus itself.

The increase of alpha3/alpha2 frequency ratio in our results support the concomitance of anterior attentive mechanism impairment in subject with MCI, even though there are not overt clinical deficits. The mayor association of the increase of alpha3/alpha2 frequency ratio with the hippocampal formation within the AHC, suggest that this filter activity is carried out by hippocampus and its input-output connections along anterior attentive circuit and AHC. Interestingly, a recent work has demonstrated that the mossy fiber (MF) pathway of the hippocampus connects the dentate gyrus to the auto-associative CA3 network, and the information it carries is controlled by a feedforward circuit combining disynaptic inhibition with monosynaptic excitation. Analysis of the MF associated circuit revealed that this circuit could act as a highpass filter (Zalay and Bardakjian, 2006).

\section{LINKING THE CHOLINERGIC HYPOTHESIS, THE HIPPOCAMPAL ATROPHY AND THE BRAIN RHYTHMS MODIFICATIONS IN SUBJECTS WITH MCI AND ALZHEIMER'S DISEASE}

Hippocampus and porencephalic cholinergic pathways are strictly connected. There are two main pathways of the hippocampal connections: the polysynaptic hippocampal pathway encompassing posterior cingulate/retrosplenial and medial temporal cortex and the direct hippocampal pathway encompassing the temporal pole, temporo-parietal association cortex, and dorsal prefrontal cortex. Recent studies have demonstrated that the polysynaptic hippocampal pathway is early affected in MCI whereas the direct pathway is affected later, in patients with AD (Frisoni et al., 2009). This pattern of damage follows some ways the cholinergic damage. Indeed, corticopetal cholinergic pathways, originating in the nucleus basalis of Meynert are affected early in the decourse of $\mathrm{AD}$. On the contrary, the damage of the cholinergic pathways that innervate directly the hippocampal formation are relatively spared at the beginning. The possible different roles of the cholinergic pathways are supported by the different effects of increasing cholinergic damage on alpha3 power band (Moretti et al., 2008b). Indeed, our results show that, when cholinergic damage becomes larger (i.e., in the group with greater cholinergic impairment) alpha3 power band increases in patients with damage in the perisylvian and medial cholinergic pathway and decreases in patients with damage in the capsular cholinergic pathway (Figure 6). This result suggests different actions for the different cholinergic pathways. The cholinergic system is thought to exert two mean functions: (1) fronto-parietal, top-down, attentional inhibitory control and (2) signal-driven, bottom-up, excitatory effect when external stimuli (via-thalamus) have to be processed (Sarter et al., 2003, 2005). For anatomical reason, being the largest tract connecting the opposite cerebral lobes, each capsular pathway is more suitable for the antero-posterior inhibitory effects and modulation of attentive functions whereas the perisylvian and medial pathways could subserve the integration of thalamus-driven stimuli in higher order cortical areas providing a common substrate for development of superior cognitive functions such as language and memory (Mesulam, 2004; Sarter et al., 2005). For anatomical reasons, perisylvian pathway is suitable to subserve language function through language areas and the medial pathway to subserve retrieval of episodic memory through medial temporal-cingulate cortex (Nestor et al., 2004). The physiological basis of alpha synchronization relies on inhibitory mechanisms (Klimesch et al., 2007). Moreover, previous works proposed that alpha3 is more involved in cortical processes than other alpha sub-bands (Klimesch, 1999; Moretti et al., 2007). Taken together, the results suggest that in the long-range connection pathway (lateral capsular) a decrease in cortical, inhibitory attentive top-down control occurs, related to a decrease in alpha3 power band. By contrast, in local, smaller, neural assemblies, like those subserved by perisylvian and medial cholinergic pathways, 


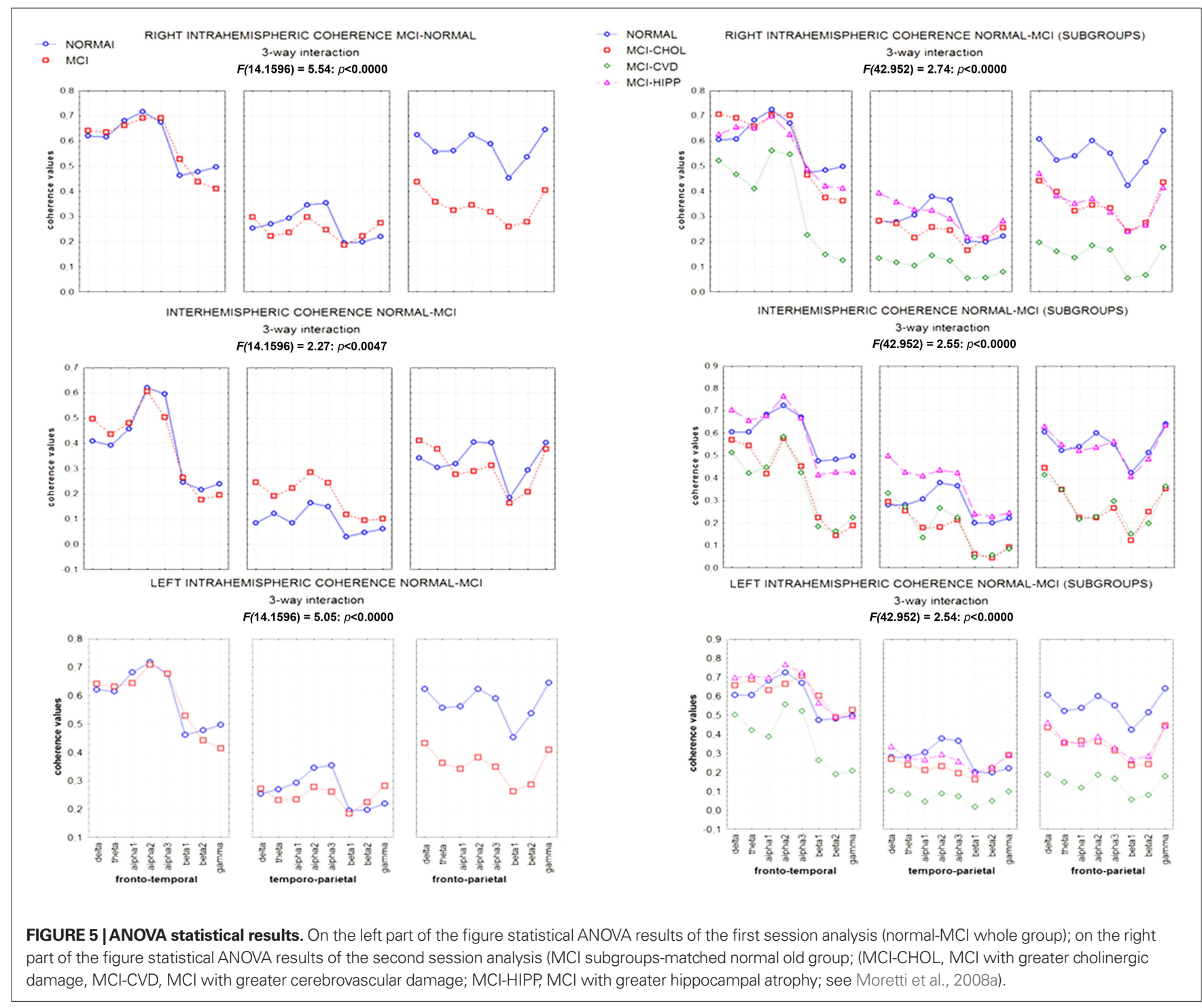

an increase of cortical inhibitory mechanism occurs, related to alpha3 power band synchronization. A reasonable explanation is that there is a removal of excitatory, synaptic inputs in intracortical networks which would produce a decrease in synaptic efficacy and functional disconnection of cortical circuits (Steriade, 2006). The dysregulation of cholinergic system could result in specific clinical symptoms according to the cholinergic pathway involved: attention for the capsular pathway, language for the perisylvian pathway, and memory for the medial pathway (Figure 5).

In this view, such process could be imagined: (1) damage of corticopetal cholinergic pathways determining an impairment of the multisynaptic hippocampal pathway. The damage of this posterior pathway induces an interruption of large brain networks and a reorganization in smaller loco-regional areas. Alpha2 rhythm is the expression of functional coupling of large brain areas whereas alpha3 suggests synchronization of smaller areas. So, the increase of alpha3/alpha2 ratio is a reasonable consequence; (2) the damage of corticopetal cholinergic pathways could induce an excitation/disinhibition of the cholinergic pathways that innervates the AHC. This cholinergic action could stimulate the direct hippocampal pathway relatively spared in the initial stage of AD. Together with the loss of inhibition mechanism, an increase of theta/gamma ratio is produced together with and increase of medial temporal functional coupling; Given that the theta rhythm generation seems more functionally linked with amygdala (Moretti et al., 2009b), with the progression of disease, and degeneration of the hippocampal direct pathway, an increase of theta/gamma ratio could be expected whereas the alpha3/alpha2 ratio is more suitable as EEG predictor of conversions from MCI state in AD (Figure 4; Moretti et al., 2009c); (3) in the later stages of $\mathrm{AD}$, with the progressive atrophy of the neural networks, both alpha3/alpha2 and theta/gamma ratio will modify according to the progression of atrophy in amygdala and/or hippocampus. Moreover, a recent study shows a correlation between brain electrical activity collected by scalp EEG and discrete mapped hippocampal areas in subjects with AD (Moretti et al., submitted; Figure 7). 


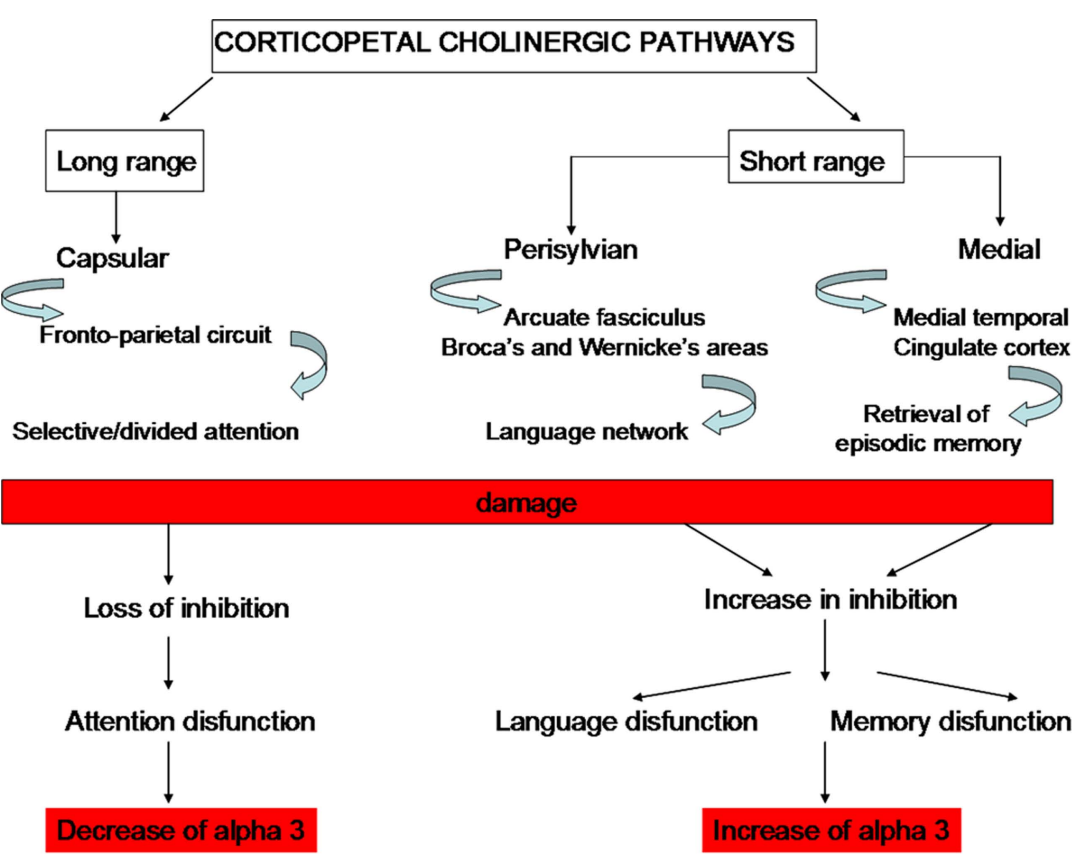

FIGURE 6 |Theoretical, neurophysiological and clinical effects of damage to the cholinergic pathways (see Moretti et al., 2008b).

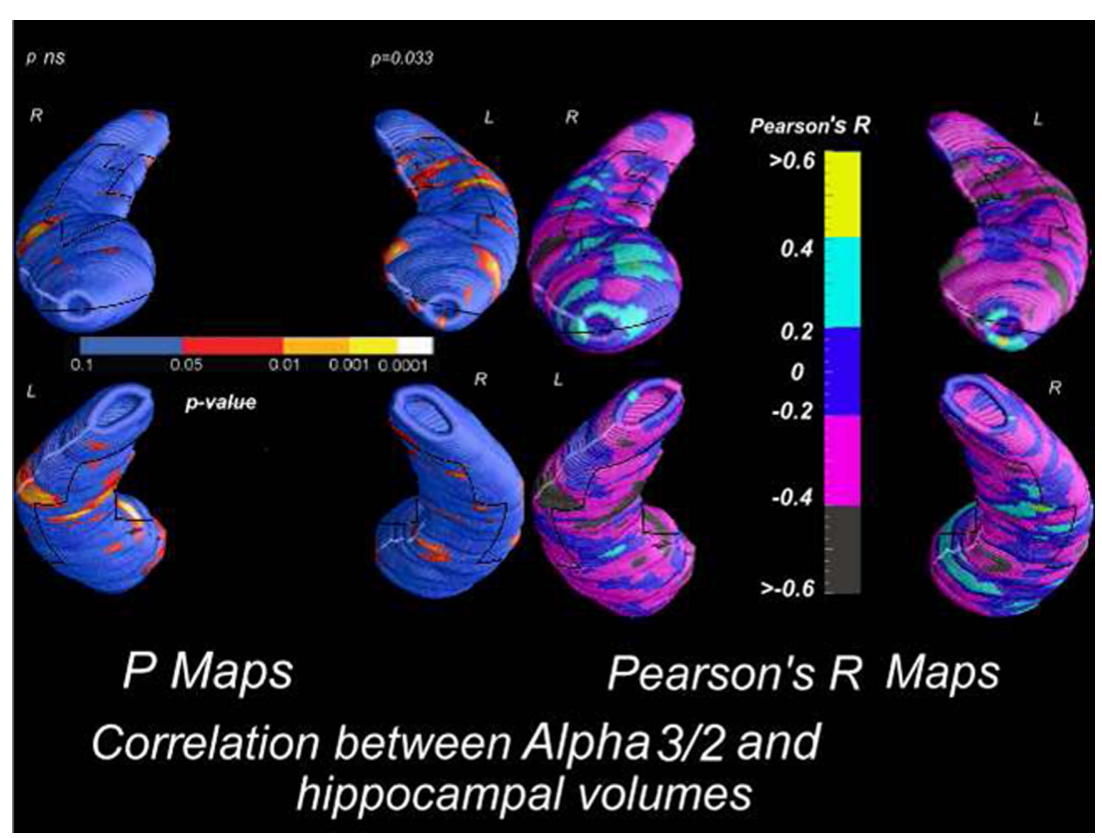

FIGURE 7 | Correlation between alpha3/alpha2 power rhythm ratio and volumes of hippocampal subregions in AD patients.

In particular, in $\mathrm{AD}$ patients the increase of both alpha3 rhythm spectral power and alpha3/alpha2 power ratio is correlated with the decrease of left hippocampal gray matter volumes. In particular, hippocampal areas involved in correlation are: presubiculum, dorsal and ventral subiculum, $\mathrm{CA} 2-\mathrm{CA} 3$ sectors of the body, CA1 mesial and lateral portion of the head. These findings confirms previous results obtained in a large cohort of patient with $\mathrm{MCI}$ who convert in $\mathrm{AD}$. Obviously, this physiological model needs further evidence supporting the relationship with the clinical symptoms. Anyway, although speculative, it could be an interesting starting point for future works. 


\section{REFERENCES}

Arnold, S. E., Hyman, B. T., Flory, J., Damasco,A.R., and Van Hoesen, G.W. (1991). The topographical and neuroanatomical distribution of neurofibrillary tangles and neuritic plaques in the cerebral cortex of patients with Alzheimer's disease. Cereb. Cortex 1, 103-116.

Babiloni, C., Babiloni, F., Carducci, F., Cincotti, F., Del Percio, C., De Pino, G., Maestrini, S., Priori, A., Tisei, P., Zanetti, O., and Rossini, P. M. (2000). Movement-related electroencephalographic reactivity in Alzheimer disease. Neuroimage 12, 139-146.

Babiloni, C., Binetti, G., Cassetta, E., Dal Forno, G., Del Percio, C., Ferreri, F., Ferri, R., Frisoni, G., Hirata, K., Lanuzza, B., Miniussi, C., Moretti, D. V., Nobili, F., Rodriguez, G., Romani, G. L., Salinari, S., and Rossini, P. M. (2006). Sources of cortical rhythms change as a function of cognitive impairment in pathological aging: a multi-centric study. Clin. Neurophysiol. $117,252-268$.

Bennett, D. A., Schneider, J. A., Bienais, J. L., Evans, D. A., and Wilson, R. S. (2004). Mild cognitive impairment is related to Alzheimer disease pathology and cerebral infarctions. Neurology 23 , 325-335.

Berardi, A. M., Parasuraman, R., and Haxby, J. V. (2005). Sustained attention in mild Alzheimer's disease. Dev. Neuropsychol. 58, 507-537.

Bobinski, M., Wegiel, J., Wisniewski, H. M., Tarnawski, M., Reisberg, B., Mlozidc, B., de Leon, M. J., and Miller, D. C. (1995). Atrophy of hippocampal formation subdivisions correlates with stage and duration of Alzheimer's disease. Dementia 6, 205-210.

Bragin, A., Jando, G., Nadasdy, Z., Hetke, J., Wise, K., and Buzsaki, G. (1995). Gamma $(40-100 \mathrm{~Hz})$ oscillation in the hippocampus of the behaving rat. $J$. Neurosci. 15, 47-60.

Callen, D. J., Black, S. E., Gao, F., Caldwell, C. B., and Szalai, J. P. (2001). Beyond the hippocampus: MRI volumetry confirms widespread limbic atrophy in AD. Neurology 57, 1669-1674.

Coburn, K. L., Lauterbach, E. C., Boutros, N. N., Black, K. J., Arciniegas, D. B., and Coffey, C. E. (2006). The value of quantitative electroencephalography in clinical psychiatry: a report by the committee on research of the American neuropsychiatric association. J. Neuropsychiatry Clin. Neurosci. 18, 460-500. Review.

De Curtis, M., and Parè, D. (2004). The rhinal cortices: a wall of inhibition between the neocortex and the hippocampus. Prog. Neurobiol. 74, 101-110.
Della Maggiore, V., Chau, W., Peres-Neto, P. R., and McIntosh, A. R. (2002). An empirical comparison of SPM preprocessing parameters to the analysis of fMRI data. Neuroimage 17, 19-28.

Dickerson, B. C., Salat, D. H., Bates, J. F., Atiya, M., Kiliiany, R. J., Greve, D. N., Dale, A. M., Stern, C. E., Blacker, D., Albert, M. S., and Sperling, R. A. (2004). Medial temporal lobe function and structure in mild cognitive impairment. Ann. Neurol. 56, 27-35.

Dickerson, B. C., Salat, D. H., Greve, D. N., Chua, E. F., Rand-Giovannetti, E., Rentz, D. M., Bertram, L., Mullin, K., Tanzi, R. E., Blacker, D., and Albert, M. S. (2005). Increased hippocampal activation in mild cognitive impairment compared to normal aging and AD. Neurology 65, 404-411.

Du, A. T., Schuff, N., Amend, D., Laasko, M. P., Hsu, Y. Y., Jagust, W. J., Yaffe, K., Kramer, J. H., Reed, B., Norman, D., Chui, H. C., and Weiner, M. W. (2001). Magnetic resonance imaging of the entorhinal cortex and hippocampus in mild cognitive impairment and Alzheimer's disease. J. Neurol. Neurosurg. Psychiatr. 71, 441-447.

Ellison, J. M., Harper, D. G., Berlow, Y., and Zeranski, L. (2008). Beyond the "C" in MCI: noncognitive symptoms in amnestic and non-amnestic mild cognitive impairment. CNS Spectr. 13, 66-72.

Ferreri, F., Pauri, F., Pasqualetti, P., Fini, R., Dal Forno, G., and Rossini, P. M. (2003). Motor cortex excitability in Alzheimer's disease: a transcranial magnetic stimulation study. Ann. Neurol. 53, 102-108.

Frisoni, G. B., Prestia, A., Rasser, P. E., Bonetti, M., and Thompson, P. M. (2009). In vivo mapping of incremental cortical atrophy from incipient to overt Alzheimer's disease. J. Neurol. 256, 916-924.

Garolera, M., Coppola, R., Muñoz, K. E., Elvevåg, B., Carver, F. W., Weinberger, D. R., and Goldberg, T. E. (2007). Amygdala activation in affective priming: a magnetoencephalogram study. Neuroreport 18, 1449-1453.

Gloveli, T., Dugladze, T., Rotstein, H. G., Traub, R. D., Monyer, H., Heinemann, U., Whittington, M. A., and Kopell, N. J. (2005). Orthogonal arrangement of rhythm-generating microcircuits in the hippocampus. Proc. Natl. Acad. Sci. U.S.A. 102, 13295-13300.

Gold, G., Bouras, C., Kovari, E., Canito, A., Glaria, B. G., Malky, A., Hof, P. R., Michel, J. P., and Giannakopoulos, P. (2000). Clinical validità of Braak neuropathological staging in the oldestold. Acta Neuropathol. 99, 579-582.

Golob, E. J., Irimajiri, R., and Starr, A. (2007). Auditory cortical activity in amnestic mild cognitive impairment: relationship to subtype and conversion to dementia. Brain 130, 740-752.

Hamalainen, A., Pihlaimaki, M., Tanila, H., Hanninen, T., Niskanen, E., Tervo, S., Karjalainen, P. A., Vanninen, R. L., and Soininen, H. (2006). Increased fMRI responses during encoding in mild cognitive impairment. Neurobiol. Aging 28, 1889-1903.

Huang, C., Wahlund, L. O., Dierks, T., Julin, P., Winblad, B., and Jelic, V. (2000). Discrimination of Alzheimer's disease and mild cognitive impairment by equivalent EEG sources: a cross-sectional and longitudinal study. Clin. Neurophysiol. 11 1961-1967.

Jelic, V., Johansson, S. E., Almkvist, O., Shigeta, M., Julin, P., Nordberg, A. Winblad, B., and Wahlund, L.O. (2000). Quantitative electroencephalography in mild cognitive impairment: longitudinal changes and possible prediction of Alzheimer's disease. Neurobiol. Aging 21, 533-540.

Jelic, V., Shigeta, M., and Julin, P. (1996). Quantitative electroencephalography power and coherence in Alzheimer's disease and mild cognitive impairment. Dementia 7, 314-323.

Jiang, Z. (2005). Study on EEG power and coherence in patients with mild cognitive impairment during working memory task. J. Zhejiang Univ. Sci. B 6, 1213-1219.

Jiang, Z., and Zheng, L. (2006). Inter and intra-hemispheric EEG coherence in patients with mild cognitive impairment at rest and during working memory task. J. Zhejiang Univ. Sci. B 5, 357-364.

Johannsen, P., Jacobsen, J., Bruhn, P., and Gjedde, A. (1999). Cortical responses to sustained and divided attention in Alzheimer's disease. Neuroimage 10, 269-281.

John, E. R., and Prichep, L. S. (1990). Neurometric studies of aging and cognitive impairment. Prog. Brain Res. 85, 555-65. Review.

John, E. R., and Prichep, L. S. (2006). The relevance of QEEG to the evaluation of behavioural disorders and pharmacological intervention. Clin. EEG Neurosci. 37, 135-143.

Johnson, J. D. (2006). The conversational brain: fronto-hippocampal interaction and disconnection. Med. Hypotheses $67,759-764$.

Klimesch, W. (1999). EEG alpha and theta oscillations reflect cognitive and memory performance: a review and analysis. Brain Res. Rev. 29, 169-195.

Klimesch, W., Sauseng, P., and Hanslmayr, S. (2007). EEG alpha oscillations: the inhibition timing hypothesis. Brain Res. Rev. 53, 63-88.
Koenig, T., Prichep, L., Dierks, T., Hubl, D. Wahlund, L.O., John, E. R., and Jelic, V. (2005). Decreased EEG synchronization in Alzheimer's disease and mild cognitive impairment. Neurobiol. Aging 26, 165-171.

Lavenex, P., and Amaral, D. G. (2000). Hippocampal-neocortical interaction: a hierarchy of associativity. Hippocampus 10, 420-430.

Levinoff, E. J., Saumier, D., and Chertkow, H. (2005). Focused attention deficits in patients with Alzheimer's disease and mild cognitive impairment. Brain Cogn. 57, 127-130.

Machulda, M. M., Ward, H. A., Borowski, B., Gunter, J. L., Cha, R. H., O’Brien, P. C., Petersen, R. C., Boeve, B. F., Knopman, D., Tang-Wai, D. F., Ivnik, R. J., Smith, G. E., Tangalos, E. G., and Jack, C. R. Jr. (2003). Comparison of memory fMRI response among normal, MCI, and Alzheimer's patients. Neurology 61, 500-506.

Mesulam, M. M. (2004). The cholinergic innervation of the human cerebral cortex. Prog. Brain Res. $145,67-78$.

Montgomery, S. M., and Buzsaki, G. (2007). Gamma oscillations dynamically couple hippocampal CA3 and $\mathrm{CA} 1$ regions during memory task performance. Proc. Natl. Acad. Sci. U.S.A. 104, 14495-14500.

Moretti, D. V., Fracassi, C., Pievani, M., Geroldi,C., Binetti, G., Zanetti,O., Sosta, K., Rossini, P. M., and Frisoni, G. B. (2009a). Increase of theta/gamma ratio is associated with memory impairment. Clin. Neurophysiol. 120, 295-303.

Moretti, D. V., Pievani, M., Fracassi, C., Binetti, G., Rosini, S., Geroldi, C., Zanetti, O., Rossini, P.M., and Frisoni, G.B. (2009b). Increase of theta/gamma and alpha3/alpha2 ratio is associated with amygdalo-hippocampal complex atrophy. J. Alzheimers Dis. 17, 349-357.

Moretti, D. V., Frisoni, G. B., Fracassi, C., Pievani, M., Geroldi, C., Binetti, G. Rossini, P. M., and Zanetti, O. (2009c). MCI patients' EEGs show group differences between those who progress and those who do not progress to AD. Neurobiol. Aging doi: 10.1016/j. neurobiolaging.2009.04.003. [Epub ahead of print].

Moretti, D. V., Frisoni, G. B., Pievani, M., Rosini, S., Geroldi, C., Binetti, G., and Rossini,P.M. (2008a). Cerebrovascular disease and hippocampal atrophy are differently linked to functional coupling of brain areas: an EEG coherence study in MCI subjects. J. Alzheimers Dis. 14, 285-299.

Moretti, D. V., Pievani, M., Fracassi, C., Geroldi, C., Calabria, M., DeCarli, C., and Rossini, P. M. (2008b). Brain vas- 
cular damage of cholinergic pathways and E.E.G. markers in mild cognitive impairment. J. Alzheimers Dis. 15, 357-372.

Moretti, D. V., Miniussi, C., Frisoni, G. B., Geroldi, C., Zanetti, O., Binetti, G., and Rossini, P. M. (2007). Hippocampal atrophy and EEG markers in subjects with mild cognitive impairment. Clin. Neurophysiol. $118,2716-2729$.

Narayanan, R. T., Seidenbecher, T., Sangha, S., Stork, O., and Pape, H. C. (2007). Theta resynchronization during reconsolidation of remote contextual fear memory. Neuroreport $18,1107-1111$.

Nestor, P. J., Scheltens, P., and Hodges, J. R. (2004). Advances in early detection of Alzheimer's disease. Nat. Med. 10, 34-41.

Paré, D., Collins, D. R., and Pelletier, J. G. (2002). Amygdala oscillations and the consolidation of emotional memories. Trends Cogn. Sci. 6, 306-314.

Pariente, J., Cole, S., Henson, R., Clare, L., Kennedy, A., Rossor, M., Cipoloti, L. Puel, M., Demonet, J.F., Chollet, F., and Frackowiak, R. S. (2005). Alzheimer's patients engage an alternative network during a memory task. Ann. Neurol. 59, 870-879.

Pijnenburg, Y. A. L., Made, Y., Knol, D. L., van Cappellen van Walsum, A. M., Knol, D. L., Scheltens, P., and Stam, C. J. (2004). EEG synchronization likelihood in mild cognitive impairment and Alzheimer's disease during a working memory task. Clin. Neurophysiol. 115, 1332-1339.

Price, J. L., and Morris, J. C. (1999). Tangles and plaques in nondemented aging and preclinical Alzheimer's disease. Ann. Neurol. 45, 358-368.

Prichep, L. S., John, E. R., Ferris, S. H., Reisberg, B., Almas, M., Alper, K., and Cancro, R. (1994). Quantitative EEG correlates of cognitive deterioration in the elderly. Neurobiol. Aging. 15, 85-90. Erratum in: Neurobiol Aging 1994 May-June; 15(3), 391.

Rozzini, L., Vicini Chilovi, B., Conti, M., Delrio, I., Borroni, B., Trabucchi, M., and Padovani, A. (2008). Neuropsychiatric symptoms in amnestic and nonamnestic mild cognitive impairment. Dement. Geriatr. Cogn. Disord. 25, 32-36.

Sarter, M., Bruno, J. P., and Givens, B. (2003). Attentional functions of cortical cholinergic inputs: what does it mean for learning and memory? Neurobiol. Learn. Mem. 80, 245-256.

Sarter, M., Hasselmo, M. E., Bruno, J. P., and Givens, B. (2005). Unraveling the attentional functions of cortical cholinergic inputs: interactions between signal-driven and cognitive modulation of signal detection. Brain Res. Rev. 48, 98-111.

Sauseng, P., Klimesch, W., Doppelmayr, M., Hanslmayr, S., Schabus, M., and Gruber, W. R. (2004). Theta coupling in the human electroencephalogram during a working memory task. Neurosci. Lett. 354, 123-126.

Schonheit, B., Zarski, R., and Ohm, T. G. (2004). Spatial and temporal relationships between plaques and tangles in Alzheimer pathology. Neurobiol. Aging 25, 697-711.

Seidenbecher, T., Laxmi, T. R., Stork, O., and Pape, H.C. (2003). Amygdalar and hippocampal theta rhythm synchroni- zation during fear memory retrieval. Science 301, 846-850.

Stam, C. J., van der Made, Y., Pijnenburg, Y. A., and Scheltens, P. (2003). EEG synchronization in mild cognitive impairment and Alzheimer's disease. Acta Neurol. Scand. 108, 90-96.

Steriade, M. (2006). Grouping of brain rhythms in corticothalamic systems. Neuroscience 137, 1087-1106.

Tales, A., Haworth, J., Nelson, S., Snowden, R. J., and Wilcock, G. (2005a). Abnormal visual search in mild cognitive impairment and Alzheimer's disease. Neurocase 11, 80-84.

Tales, A., Snowden, R. J., Haworth, J., and Wilcock, G. (2005b). Abnormal spatial and non-spatial cueing effects in mild cognitive impairment and Alzheimer's disease. Neurocase 11, 85-92.

Vecchio, F., Babiloni, C., Ferreri, F., Curcio, G., Fini, R., Del Percio, C., and Rossini, P. M. (2007). Mobile phone emission modulates interhemispheric functional coupling of EEG alpha rhythms. Eur. J. Neurosci. 25, 1908-1913.

Young, K. A., Holcomb, L. A., Bonkale, W. L., Hicks, P. B., Yazdani, U., and German, D. C. (2007). 5HTTLPR polymorphism and enlargement of the pulvinar: unlocking the backdoor to the limbic system. Biol. Psychiatry 61, 813-818.

Zalay, O. C., and Bardakjian, B. L. (2006). Simulated mossy fiber associated feedforward circuit functioning as a highpass filter. Conf. Proc. IEEE Eng. Med. Biol. Soc. 1, 4979-4982.

Zappoli, R., Versari, A., Paganini, M., Arnetoli, G., Muscas, G. C., Gagmen, P. F., Arneodo, M. G., Poggiolini, D.,
Zappoli, F., and Battaglia, A. (1995). Brain electrical activity (quantitative EEG and bit-mapping neurocognitive CNV components), psychometrics and clinical findings in presenile subjects with initial mild cognitive decline or probable Alzheimer-type dementia. Ital. J. Neurol. Sci. 16, 341-376.

Zheng, L., Jiang, Z., and Yu, E. (2007). Alpha spectral power and coherence in the patients with mild cognitive impairment during a three-level working memory task. J. Zhejiang Univ. Sci. B 8, 584-592.

Conflict of Interest Statement: The authors declare that the research was conducted in the absence of any commercial or financial relationships that could be construed as a potential conflict of interest.

Received: 12 June 2010; accepted: 17 December 2010; published online: 04 January 2011.

Citation: Moretti DV, Frisoni GB, Binetti $G$ and Zanetti $O$ (2011) Anatomical substrate and scalp EEG markers are correlated in subjects with cognitive impairment and Alzheimer's disease. Front. Psychiatry 1:152. doi: 10.3389/fpsyt.2010.00152

This article was submitted to Frontiers in Neurodegeneration, a specialty of Frontiers in Psychiatry.

Copyright (C) 2011 Moretti, Frisoni, Binetti and Zanetti. This is an open-access article subject to an exclusive license agreement between the authors and the Frontiers Research Foundation, which permits unrestricted use, distribution, and reproduction in any medium, provided the original authors and source are credited. 